\title{
Non-vanishing of modular $L$-functions on a disc
}

\author{
by
}

\author{
Amir Akbary (Montréal)
}

Introduction. Non-vanishing of $L$-functions on a disc has been studied in various contexts in the recent years. In the context of Dirichlet $L$-functions P. Elliott [6] proved that there are infinitely many Dirichlet $L$-functions $L\left(s, \chi_{p}\right)\left(\chi_{p}\right.$ is a Dirichlet character mod $p$ (prime)) which are uniformly bounded below by $c(\log p)^{1 / 2}$ in the disc $|s-1 / 2| \leq(\log p)^{-(1+\varepsilon)}$, and so do not vanish there. This result has been improved by R. Balasubramanian in [2]. He proved that the number of Dirichlet $L$-functions $L\left(s, \chi_{p}\right)$ that do not vanish in the disc $|s-1 / 2| \leq(\log p)^{-(1+\varepsilon)}$ is bounded below by $c p(\log p)^{-2}$. Also, in [3] R. Balasubramanian and K. Murty studied nonvanishing of Dirichlet $L$-functions in the disc $\left|s-\sigma_{j}\right| \leq 2(\log p)^{-1}$, where $\sigma_{j}=1 / 2+j / \log p$ and $2 \leq j \leq(\log p) / 2-2$. They proved that for a positive proportion of the characters $\chi_{p}(\bmod p), L\left(s, \chi_{p}\right)$ does not have a real zero in the region $1 / 2+c / \log p \leq \operatorname{Re}(s)<1$. Here, $c>0$ is an absolute constant and $p$ is a sufficiently large prime.

In this paper we prove an analogue of the above results in the context of modular $L$-functions. We are interested in the zeros of $L_{f}(s, \chi)$ in the critical strip $k / 2<\operatorname{Re}(s)<(k+1) / 2$, where $L_{f}(s, \chi)$ is the twisted $L$-function associated with the newform $f$ and Dirichlet character $\chi$. Generalized Riemann Hypothesis predicts that $L_{f}(s, \chi)$ is non-zero in this strip. One of the known results in the subject is given by K. Murty and T. Stefanicki [7]. They proved that at least $Y^{2 / 3-\varepsilon}$ quadratic twists $L_{f}\left(s, \chi_{d}\right)(|d| \leq Y, d \equiv 1$ $(\bmod 4))$ attached to holomorphic newforms and $Y^{2 / 3-\varepsilon}$ attached to Maass newforms do not vanish inside the disc $\left|s-s_{0}\right|<(\log Y)^{-(1+\varepsilon)}$ for any $\varepsilon>0$ and any point $s_{0}$ inside the critical strip (the exponent $2 / 3$ can in fact be improved now to 1 using improved character sum estimates of Heath-Brown as in the work of Perelli and Pomykała [8]).

Here, we prove the following theorem.

2000 Mathematics Subject Classification: Primary 11F67.

Research partially supported by Concordia Research Funds. 
THEOREM 1. Let $s_{0}=\sigma_{0}+i t_{0}$ be a point in the strip $k / 2<\operatorname{Re}(s)<$ $(k+1) / 2$ and let $C_{N}$ be the disc with center $s_{0}$ and radius $r_{N}=o(1)$ (i.e. $r_{N} \rightarrow 0$ as $\left.N \rightarrow \infty\right)$. Suppose that $\chi$ is a fixed primitive Dirichlet character mod $q$ such that $(q, N)=1$. Then there are positive constants $C_{\sigma_{0}, k}$ (depending only on $k$ and $\sigma_{0}$ ) and $C_{s_{0}, q, k, r_{N}}$ (depending on $q, k, s_{0}$ and $r_{N}$ ) such that for prime $N>C_{s_{0}, q, k, r_{N}}$ there exist at least $C_{\sigma_{0}, k} N(\log N)^{-1}$ newforms $f$ of weight $k$ and level $N$ for which $L_{f}(s, \chi) \neq 0$ for all $s \in C_{N}$.

The methodology of the proof is based on a comparison of mean values. In Sections 3 and 4 , we derive asymptotic formulae for $L_{f}\left(s_{f}, \chi\right)$ and $\left|L_{f}\left(s_{f}, \chi\right)\right|^{2}$ on average, where $s_{f}$ is an arbitrary point in the disc $C_{N}$. To do this first we derive the asymptotic formulae for a fixed point $s_{0}$ in the critical strip (Lemmas 5 and 7). These are analogues of the results given by W. Duke [4] for the center of critical strip. Then an application of Cauchy's integral formula gives us the asymptotic formulae on a disc (Propositions 1 and 2). This technique has already been applied by P. Elliott, B. Balasubramanian and B. Balasubramanian-K. Murty for Dirichlet $L$-functions. Finally we have to deal with the contribution of oldforms; we apply the technique developed by the author in [1] to overcome this difficulty. In Section 5 we finish off the proof of Theorem 1 by an application of the Cauchy-Schwarz inequality.

Finally, with a slight modification of our previous results, we establish asymptotic formulae for $L_{f}\left(s_{f}, \chi\right)$ and $\left|L_{f}\left(s_{f}, \chi\right)\right|^{2}$ on average, where $s_{f}$ is an arbitrary point in the $\operatorname{disc} C_{N}$ with center on the critical line $s=k / 2+i t$, and as a result we prove the following non-vanishing theorem.

Theorem 2. Let $s_{0}=k / 2+i t_{0}$ and let $C_{N}$ be the disc with center $s_{0}$ and radius $r_{N}=1 /(\log N)^{4+\varepsilon}(\varepsilon>0)$. Suppose that $\chi$ is a fixed primitive Dirichlet character mod $q$ such that $(q, N)=1$. Then there are positive constants $C_{k}$ (depending only on $k$ ) and $C_{t_{0}, q, k, \varepsilon}$ (depending on $q, k, t_{0}$ and $\varepsilon)$ such that for prime $N>C_{t_{0}, q, k, \varepsilon}$ there exists at least $C_{k} N(\log N)^{-2}$ newforms $f$ of weight $k$ and level $N$ for which $L_{f}(s, \chi) \neq 0$ for all $s \in C_{N}$.

Acknowledgements. The author would like to thank Kumar Murty and the referee for reading the manuscript and providing many valuable suggestions.

2. Preliminaries. In this section we review some basic facts concerning modular forms and set up our notation.

Let $S_{k}(N)$ be the space of cusp forms of weight $k$ for $\Gamma_{0}(N)$ with trivial character. The space $S_{k}(N)$ has an inner product (Petersson inner product)

$$
\langle f, g\rangle=\int_{\Gamma_{0}(N) \backslash \mathcal{H}} f(z) \overline{g(z)} y^{k} \frac{d x d y}{y^{2}}
$$


where $\mathcal{H}$ denotes the upper half-plane. For any $f \in S_{k}(N)$ let

$$
f(z)=\sum_{n=1}^{\infty} a_{f}(n) e(n z), \quad e(z)=e^{2 \pi i z},
$$

be the Fourier expansion of $f$ at $i \infty$.

Let $\chi$ be a primitive Dirichlet character $\bmod q$ with $(q, N)=1$. Then the twisted L-function associated with $f$ and $\chi$ is defined by

$$
L_{f}(s, \chi)=\sum_{n=1}^{\infty} \frac{\chi(n) a_{f}(n)}{n^{s}} .
$$

The twisted $L$-function is given by an absolutely convergent series on the half-plane $\operatorname{Re}(s)>(k+1) / 2$ and it has an analytic continuation to the whole plane. Moreover, if $f$ is a newform (in Atkin-Lehner sense), then $L_{f}(s, \chi)$ has an Euler product valid on $\operatorname{Re}(s)>(k+1) / 2$ and it satisfies the following functional equation:

$$
\left(\frac{q \sqrt{N}}{2 \pi}\right)^{s} \Gamma(s) L_{f}(s, \chi)=\varepsilon_{\chi}\left(\frac{q \sqrt{N}}{2 \pi}\right)^{k-s} \Gamma(k-s) L_{f}(k-s, \bar{\chi})
$$

where $\varepsilon_{\chi}=\varepsilon_{f} \chi(N) \tau(\chi)^{2} q^{-1}$ with $\varepsilon_{f}= \pm 1$ (the root number of $f$ ) which depends only on $f$ and $\tau(\chi)$ is the Gauss sum.

Let $\left\{T_{p}(p \nmid N), U_{q}(q \mid N)\right\}$ be the collection of the classical Hecke operators and let $W_{q}(q \mid N)$ be the "W operator" of Atkin and Lehner. In 1983 A. Pizer introduced the operators $C_{q}$ on $S_{k}(N)$ for $q \mid N$, such that the action of $C_{q}$ on the new part of $S_{k}(N)$ is the same as the action of the classical $U_{q}$ operators. More precisely he defined $C_{q}$ as

$$
C_{q}= \begin{cases}U_{q}+W_{q} U_{q} W_{q}+q^{k / 2-1} W_{q} & \text { if } q \| N \\ U_{q}+W_{q} U_{q} W_{q} & \text { if } q^{2} \mid N .\end{cases}
$$

Then he showed that $T_{p}(p \nmid N), C_{q}(q \mid N)$ form a commuting family of Hermitian operators. Using this, he proved ([9], Theorem 3.10) the following result:

THEOREM. There exists a basis $f_{i}(z)\left(1 \leq i \leq \operatorname{dim} S_{k}(N)\right)$ of $S_{k}(N)$ such that each $f_{i}(z)$ is an eigenform for all the $T_{p}$ and $C_{q}$ operators with $p \nmid N$ and $q \mid N$. Let $f(z)=\sum_{n=1}^{\infty} a_{f}(n) e(z)$ be an element of this basis. Then $a_{f}(1) \neq 0$ and assuming $f(z)$ is normalized so that $a_{f}(1)=1$, we have $f \mid T_{p}=a_{f}(p) f$ for all $p \nmid N, f \mid C_{q}=a_{f}(q) f$ for all $q \mid N$, and $a_{f}(n m)=$ $a_{f}(n) a_{f}(m)$ whenever $(n, m)=1$. Furthermore $f(z)$ is an eigenform for all $W_{q}$ operators, $q \mid N$. Finally, if $g(z) \in S_{k}(N)$ is an eigenform for all the $T_{p}$ and $C_{q}$ operators with $p \nmid N$ and $q \mid N$, then $g(z)=c f_{i}(z)$ for some $c \in \mathbb{C}^{*}$ and some unique $i, 1 \leq i \leq \operatorname{dim} S_{k}(N)$. 
Now let $\mathcal{F}_{N}$ be the set of all normalized $\left(a_{f}(1)=1\right)$ newforms in $S_{k}(N)$ and let $\mathcal{P}_{N}$ be the basis of $S_{k}(N)$ given by the above theorem. The elements of $\mathcal{P}_{N}$ form an orthogonal basis (with respect to the Petersson inner product) for $S_{k}(N)$, any $f \in \mathcal{P}_{N}$ has real Fourier coefficient and $L_{f}(s, \chi)$ satisfies the functional equations (1). Moreover, we can show that the action of $C_{q}$ on $S_{k}(N)^{\text {new }}$ is the same as the action of $U_{q}$ (see [9], Remark 2.9). This shows that $\mathcal{F}_{N} \subset \mathcal{P}_{N}$.

For the Fourier coefficients of a newform $f$ we have the Deligne bound

$$
\left|a_{f}(n)\right| \leq \mathbf{d}(n) n^{(k-1) / 2}
$$

where $\mathbf{d}(n)$ is the divisor function. For $N$ prime, we have the following estimation of the Fourier coefficients of $f \in \mathcal{P}_{N}$.

Lemma 1. Suppose $N$ is prime and $f \in \mathcal{P}_{N}$. Then

$$
\left|a_{f}(n)\right| \leq c_{0} n^{k / 2}
$$

where $c_{0}$ is an absolute constant independent of $f$.

Pr o of. Propositions 3.6 and 3.4 of [9] imply that if $f \in \mathcal{P}_{N}-\mathcal{F}_{N}$, then

$$
f(z)=h(z) \pm N^{k / 2} h(N z)
$$

where $h$ is the normalized newform of weight $k$ and level 1 associated with $f$. Now the result follows from the Deligne bound for the newforms (see [1], Lemma 2.2, for the details).

Finally, since $\mathcal{P}_{N}$ forms an orthogonal basis of $S_{k}(N)$, the Fourier coefficients of its elements are semi-orthogonal in the following sense:

Lemma 2. Let $\omega_{f}=\Gamma(k-1) /\left((4 \pi)^{k-1}\langle f, f\rangle\right)$ and let $\delta_{m, n}$ be the Kronecker delta. For $m$ and $n$ positive integers we have the inequality

$$
\left|\sum_{f \in \mathcal{P}_{N}} \omega_{f} \frac{a_{f}(m)}{\sqrt{m^{k-1}}} \cdot \frac{a_{f}(n)}{\sqrt{n^{k-1}}}-\delta_{m, n}\right| \leq M \mathbf{d}(N) N^{1 / 2-k}(m, n)^{1 / 2} \sqrt{(m n)^{k-1}}
$$

where $M$ is a constant depending only on $k$ and $\mathbf{d}(N)$ is the number of divisors of $N$.

Proof. See [4], Lemma 1.

3. Mean estimation. In this section we will find an asymptotic formula for

$$
\sum_{f \in \mathcal{P}_{N}} \omega_{f} L_{f}\left(s_{f}, \chi\right)
$$

where $s_{f}$ is a variable point in the disc with center $s_{0}=\sigma_{0}+i t_{0}\left(k / 2<\sigma_{0}\right.$ $<(k+1) / 2)$ and radius $r_{N}=o(1)$. 
Lemma 3. For any $x>0$ and $s_{0}=\sigma_{0}+i t_{0} \in \mathbb{C}$ where $(k-1) / 2 \leq \sigma_{0} \leq$ $(k+1) / 2$, let

$$
W\left(s_{0}, x\right)=\frac{1}{2 \pi i} \int_{(5 / 4)} \Gamma\left(s+s_{0}\right) x^{-s} \frac{d s}{s}
$$

and

$$
\mathcal{A}_{f, \chi}\left(x, s_{0}\right)=\sum_{n \geq 1} \chi(n) a_{f}(n) n^{-s_{0}} W\left(s_{0}, 2 \pi n / x\right)
$$

where $\chi$ is a fixed primitive Dirichlet character $\bmod q$ with $(q, N)=1$. Then

$$
\Gamma\left(s_{0}\right) L_{f}\left(s_{0}, \chi\right)=\mathcal{A}_{f, \chi}\left(x, s_{0}\right)+\varepsilon_{\chi}\left(\frac{q \sqrt{N}}{2 \pi}\right)^{k-2 s_{0}} \mathcal{A}_{f, \bar{\chi}}\left(\frac{q^{2} N}{x}, k-s_{0}\right)
$$

where $\varepsilon_{\chi}$ is the root number of $L_{f}(s, \chi)$.

Proof. From the definition of $W\left(s_{0}, x\right)$ it is clear that

$$
\mathcal{A}_{f, \chi}\left(x, s_{0}\right)=\frac{1}{2 \pi i} \int_{(5 / 4)} L_{f}\left(s+s_{0}, \chi\right)\left(\frac{x}{2 \pi}\right)^{s} \Gamma\left(s+s_{0}\right) \frac{d s}{s} .
$$

Changing the line of integration from $5 / 4$ to $-5 / 4$ and using the functional equation (1) yields

$$
\begin{aligned}
& \mathcal{A}_{f, \chi}\left(x, s_{0}\right)=\Gamma\left(s_{0}\right) L_{f}\left(s_{0}, \chi\right) \\
& \quad+\varepsilon_{\chi}\left(\frac{q \sqrt{N}}{2 \pi}\right)^{k-2 s_{0}} \frac{1}{2 \pi i} \int_{(-5 / 4)} L_{f}\left(k-s-s_{0}, \bar{\chi}\right)\left(\frac{2 \pi x}{q^{2} N}\right)^{s} \Gamma\left(k-s-s_{0}\right) \frac{d s}{s} .
\end{aligned}
$$

Now changing variables $s \mapsto-s$ implies the result.

LEMMA 4. Under the assumptions of Lemma 3,

$$
\begin{array}{ll}
W\left(s_{0}, x\right) \ll x^{\sigma_{0}-1} e^{-x} & \text { as } x \rightarrow \infty, \\
W\left(s_{0}, x\right) \ll_{k} 1 & \text { as } x \rightarrow 0 .
\end{array}
$$

Proof. We have

$$
W\left(s_{0}, x\right)=\frac{1}{2 \pi i} \int_{(5 / 4)}\left(\int_{0}^{\infty} e^{-t} t^{s+s_{0}-1} d t\right) x^{-s} \frac{d s}{s}=\int_{x}^{\infty} t^{s_{0}-1} e^{-t} d t .
$$

Therefore

$$
\left|W\left(s_{0}, x\right)\right|=\left|\int_{x}^{\infty} t^{s_{0}-1} e^{-t} d t\right| \leq \int_{x}^{\infty} t^{\sigma_{0}-1} e^{-t} d t .
$$

Now the first result follows from the estimation of the last integral using integration by parts. The second result is clear since $\left|W\left(s_{0}, x\right)\right| \leq \Gamma\left(\sigma_{0}\right)$ as $x \rightarrow 0$. 
Lemma 5. Let $\chi$ be a fixed primitive Dirichlet character mod $q$ with $(q, N)=1$ and let $s_{0}=\sigma_{0}+i t_{0}$ be a point in the strip $(k-1) / 2<\operatorname{Re}(s) \leq$ $(k+1) / 2$. Then

$$
\begin{aligned}
\sum_{f \in \mathcal{P}_{N}} \omega_{f} L_{f}\left(s_{0}, \chi\right)=1 & +O\left(\frac{1}{\left|\Gamma\left(s_{0}\right)\right|} N^{1 / 2-\sigma_{0}}(\log N)^{k-\sigma_{0}}\right) \\
& +O\left(\frac{1}{\left|\Gamma\left(s_{0}\right)\right|} N^{(k-12) / 2-\sigma_{0}}(\log N)^{k-\sigma_{0}-1}\right)
\end{aligned}
$$

for $N$ prime. The implied constant depends only on $q$ and $k$.

Pr o o f. Choosing $x=q^{2} N \log N$ in Lemma 3 gives

$$
\mathcal{A}_{f, \bar{\chi}}\left(\frac{N q^{2}}{x}, k-s_{0}\right)=\sum_{n \geq 1} \bar{\chi}(n) a_{f}(n) n^{s_{0}-k} W\left(k-s_{0}, 2 \pi n \log N\right) .
$$

Using Lemmas 4 and 1 we have

$$
\begin{aligned}
\left|\mathcal{A}_{f, \bar{\chi}}\left(\frac{1}{\log N}, k-s_{0}\right)\right| & \leq \sum_{n \geq 1}\left|a_{f}(n)\right| n^{\sigma_{0}-k}\left|W\left(k-s_{0}, 2 \pi n \log N\right)\right| \\
& \leq \sum_{n \geq 1} c_{0} n^{k / 2} n^{\sigma_{0}-k}(2 \pi n \log N)^{k-\sigma_{0}-1} e^{-2 \pi n \log N} \\
& =c_{0}(2 \pi \log N)^{k-\sigma_{0}-1} \sum_{n \geq 1} \frac{n^{k / 2-1}}{\left(N^{2 \pi}\right)^{n}} .
\end{aligned}
$$

Therefore from Lemma 3 we get

$$
\begin{aligned}
\Gamma\left(s_{0}\right) \sum_{f \in \mathcal{P}_{N}} \omega_{f} L_{f}\left(s_{0}, \chi\right)= & \sum_{f \in \mathcal{P}_{N}} \omega_{f} \mathcal{A}_{f, \chi}\left(x, s_{0}\right) \\
& +\left(\sum_{f \in \mathcal{P}_{N}} \omega_{f}\right) O_{q, k}\left(N^{-6+k / 2-\sigma_{0}}(\log N)^{k-\sigma_{0}-1}\right) .
\end{aligned}
$$

From this, we have

$$
\begin{aligned}
\Gamma\left(s_{0}\right) & \sum_{f \in \mathcal{P}_{N}} \omega_{f} L_{f}\left(s_{0}, \chi\right)-\Gamma\left(s_{0}\right) \\
= & \sum_{n \geq 1} \chi(n)\left(\sum_{f \in \mathcal{P}_{N}} \omega_{f} \frac{a_{f}(n)}{n^{(k-1) / 2}}-\delta_{1, n}\right) W\left(s_{0}, \frac{2 \pi n}{q^{2} N \log N}\right) n^{(k-1) / 2-s_{0}} \\
& +W\left(s_{0}, \frac{2 \pi}{q^{2} N \log N}\right)-\Gamma\left(s_{0}\right) \\
& +\left(\sum_{f \in \mathcal{P}_{N}} \omega_{f}\right) O_{q, k}\left(N^{-6+k / 2-\sigma_{0}}(\log N)^{k-\sigma_{0}-1}\right) .
\end{aligned}
$$


Note that

$$
\begin{aligned}
W\left(s_{0}, \frac{2 \pi}{q^{2} N \log N}\right)-\Gamma\left(s_{0}\right) & =\int_{0}^{2 \pi /\left(q^{2} N \log N\right)} t^{s_{0}-1} e^{-t} d t \\
& =O_{q, k}\left((N \log N)^{-\sigma_{0}}\right) .
\end{aligned}
$$

Also, from Lemma 2 for $m=n=1$ it follows that

$$
\sum_{f \in \mathcal{P}_{N}} \omega_{f}=1+O\left(N^{1 / 2-k}\right)
$$

By applying $m=1$ in Lemma 2 and using the above identities, we have

$$
\begin{aligned}
& \left|\Gamma\left(s_{0}\right)\left(\sum_{f \in \mathcal{P}_{N}} \omega_{f} L_{f}\left(s_{0}, \chi\right)-1\right)\right| \\
& \leq M_{1} \frac{N^{1 / 2-k}}{(N \log N)^{\sigma_{0}-1}} \sum_{n \geq 1} n^{k-2} e^{-2 \pi n /\left(q^{2} N \log N\right)}+M_{2}(N \log N)^{-\sigma_{0}} \\
& \quad+M_{3} N^{-6+k / 2-\sigma_{0}}(\log N)^{k-\sigma_{0}-1}
\end{aligned}
$$

where $M_{1}, M_{2}$ and $M_{3}$ are constants depending on $q$ and $k$. This proves the desired result.

Proposition 1. Let $s_{0}=\sigma_{0}+i t_{0}$ be a point in the strip $k / 2<\operatorname{Re}(s)<$ $(k+1) / 2$ and let $\Gamma$ and $C_{N}$ be the circles with center $\left(\sigma_{0}, t_{0}\right)$ and radius $R=\frac{1}{2} \min \left\{(k+1) / 2-\sigma_{0}, \sigma_{0}-k / 2\right\}$ and $r_{N}=o(1)$ respectively. Then for $N$ prime

$$
\sum_{f \in \mathcal{P}_{N}} \omega_{f} L_{f}\left(s_{f}, \chi\right)=1+O_{q, k}\left(\frac{1}{\left|\Gamma\left(s_{0}\right)\right|} N^{-1 / 2}\right)+O_{q, k, s_{0}}\left(\frac{r_{N}}{R-r_{N}} N^{-1 / 2}\right)
$$

where $s_{f}$ is an arbitrary point in $C_{N}$.

Proof. By Cauchy's integral formula for any $s_{f} \in C_{N}$, we have

$$
L_{f}\left(s_{f}, \chi\right)-L_{f}\left(s_{0}, \chi\right)=\frac{1}{2 \pi i} \int_{\Gamma} L_{f}(w, \chi)\left(\frac{1}{w-s_{f}}-\frac{1}{w-s_{0}}\right) d w
$$

where $\Gamma$ is traversed in the counter clockwise direction. Therefore

$$
\text { (2) } \begin{aligned}
\sum_{f \in \mathcal{P}_{N}} \omega_{f} L_{f}\left(s_{f}, \chi\right)= & \sum_{f \in \mathcal{P}_{N}} \omega_{f} L_{f}\left(s_{0}, \chi\right) \\
& +\frac{1}{2 \pi i} \int_{\Gamma}\left(\sum_{f \in \mathcal{P}_{N}} \omega_{f} L_{f}(w, \chi)\right) \frac{s_{f}-s_{0}}{\left(w-s_{f}\right)\left(w-s_{0}\right)} d w .
\end{aligned}
$$

Now using Lemma 5 yields 
(3) $\quad\left|\frac{1}{2 \pi i} \int_{\Gamma}\left(\sum_{f \in \mathcal{P}_{N}} \omega_{f} L_{f}(w, \chi)\right) \frac{s_{f}-s_{0}}{\left(w-s_{f}\right)\left(w-s_{0}\right)} d w\right|$

$$
\leq \frac{r_{N}}{R-r_{N}} O_{q, k, s_{0}}\left(N^{-1 / 2}\right) .
$$

Note that here we used the fact that

$$
\frac{1}{2 \pi i} \int_{\Gamma} \frac{s_{f}-s_{0}}{\left(w-s_{f}\right)\left(w-s_{0}\right)} d w=0 .
$$

Applying (3) and Lemma 5 in (2) completes the proof.

4. Mean square estimation. In this section we are going to find an asymptotic formula for the average values of $\left|L_{f}\left(s_{f}, \chi\right)\right|^{2}$ where $s_{f}$ is a variable point in a disc with center $s_{0}=\sigma_{0}+i t_{0}\left(k / 2<\sigma_{0}<(k+1) / 2\right)$ and radius $r_{N}=o(1)$. We start with writing $\left|L_{f}\left(s_{0}, \chi\right)\right|^{2}$ as a sum of two convergent series.

Let $\left|L_{f}\left(s_{0}, \chi\right)\right|^{2}=\sum_{l \geq 1} b_{f}(l) l^{-\sigma_{0}}$ so that

$$
b_{f}(l)=\sum_{m n=l} \chi(n) \bar{\chi}(m) a_{f}(n) a_{f}(m)\left(\frac{m}{n}\right)^{i t_{0}} .
$$

For $x>0$ and $s_{0}=\sigma_{0}+i t_{0} \in \mathbb{C}$ where $(k-1) / 2 \leq \sigma_{0} \leq(k+1) / 2$, define

$$
\mathcal{B}_{f}\left(x, s_{0}\right)=\sum_{l \geq 1} \frac{b_{f}(l)}{l^{\sigma_{0}}} Z\left(s_{0}, l / x\right)
$$

where

$$
Z\left(s_{0}, x\right)=\frac{1}{2 \pi i} \int_{(5 / 4)}(2 \pi)^{-2 s} \Gamma\left(s+s_{0}\right) \Gamma\left(s+\bar{s}_{0}\right) x^{-s} \frac{d s}{s} .
$$

Using Deligne's bound in (4) and standard estimates for $Z\left(s_{0}, x\right)$ shows that (5) is absolutely convergent.

Lemma 6. Let $f \in \mathcal{P}_{N}$ and suppose that $\chi$ is a primitive Dirichlet character mod $q$ with $(q, N)=1$. For any $x>0$ we have

$$
\left|\Gamma\left(s_{0}\right) L_{f}\left(s_{0}, \chi\right)\right|^{2}=\mathcal{B}_{f}\left(x, s_{0}\right)+\left(\frac{q^{2} N}{4 \pi^{2}}\right)^{k-2 \sigma_{0}} \mathcal{B}_{f}\left(\frac{\left(q^{2} N\right)^{2}}{x}, k-\bar{s}_{0}\right) .
$$

Pr o of. From (6) we have

$$
\mathcal{B}_{f}\left(x, s_{0}\right)=\frac{1}{2 \pi i} \int_{(5 / 4)}(2 \pi)^{-2 s} \Gamma\left(s+s_{0}\right) \Gamma\left(s+\bar{s}_{0}\right) L_{f}\left(s+s_{0}, \chi\right) L_{f}\left(s+\bar{s}_{0}, \bar{\chi}\right) x^{s} \frac{d s}{s} .
$$

By changing the line of integration from $5 / 4$ to $-5 / 4$ and using the functional 
equation (1) we get

$$
\begin{aligned}
\mathcal{B}_{f}\left(x, s_{0}\right)= & \left|\Gamma\left(s_{0}\right) L_{f}\left(s_{0}, \chi\right)\right|^{2} \\
& +\left(\frac{q^{2} N}{4 \pi^{2}}\right)^{k-2 \sigma_{0}} \int_{(-5 / 4)}(2 \pi)^{2 s} \Gamma\left(k-s-s_{0}\right) \Gamma\left(k-s-\bar{s}_{0}\right) \\
& \times L_{f}\left(k-s-s_{0}, \bar{\chi}\right) L_{f}\left(k-s-\bar{s}_{0}, \chi\right)\left(\frac{x}{\left(q^{2} N\right)^{2}}\right)^{s} \frac{d s}{s} .
\end{aligned}
$$

Now changing variables $s \mapsto-s$ yields the result.

We estimate $\mathcal{B}_{f}\left(x, s_{0}\right)$ on average. From (4) and (5) it follows that

$$
\begin{aligned}
\sum_{f \in \mathcal{P}_{N}} \omega_{f} \mathcal{B}_{f}\left(x, s_{0}\right)= & \sum_{f \in \mathcal{P}_{N}} \omega_{f} \sum_{l \geq 1} b_{f}(l) l^{-\sigma_{0}} Z\left(s_{0}, l / x\right) \\
= & \sum_{m, n \geq 1} \chi(n) \bar{\chi}(m) Z\left(s_{0}, m n / x\right) \frac{\left(\frac{m}{n}\right)^{i t_{0}}}{(m n)^{\sigma_{0}-(k-1) / 2}} \\
& \times \sum_{f \in \mathcal{P}_{N}} \omega_{f} \frac{a_{f}(m)}{\sqrt{m^{k-1}}} \cdot \frac{a_{f}(n)}{\sqrt{n^{k-1}}} \\
= & \sum_{n \geq 1}|\chi(n)|^{2} Z\left(s_{0}, n^{2} / x\right) \frac{1}{n^{2 \sigma_{0}-k+1}}+R
\end{aligned}
$$

where

$$
R \ll N^{1 / 2-k} \sum_{m, n \geq 1} Z\left(\sigma_{0}, m n / x\right)(m, n)^{1 / 2}(m n)^{-\sigma_{0}+k-1} .
$$

Note that here we are using the inequality $\left|Z\left(s_{0}, x\right)\right| \leq Z\left(\sigma_{0}, x\right)$. This is true since by writing $\Gamma$ functions in terms of integrals in (6) and interchanging the order of integration, we have

$$
Z\left(s_{0}, x\right)=\int_{0}^{\infty} t_{1}^{s_{0}-1} e^{-t_{1}}\left(\int_{4 \pi^{2} x / t_{1}}^{\infty} e^{-t_{2}} t_{2}^{\bar{s}_{0}-1} d t_{2}\right) d t_{1} .
$$

Applying the triangle inequality in the above identity implies the desired inequality.

Using the definition of $Z\left(s_{0}, x\right)$, the first term in (7) is equal to

$$
\frac{1}{2 \pi i} \int_{(5 / 4)} L\left(2 s+2 \sigma_{0}-k+1, \chi_{0}\right)(2 \pi)^{-2 s} \Gamma\left(s+s_{0}\right) \Gamma\left(s+\bar{s}_{0}\right) x^{s} \frac{d s}{s}
$$

where $\chi_{0}$ is the principal character $\bmod q$ and $L\left(s, \chi_{0}\right)=\zeta(s) \prod_{p \mid q}\left(1-1 / p^{s}\right)$. Now we assume that $\sigma_{0} \neq k / 2$, since the integrand has simple poles at $s=0$ 
and $s=k / 2-\sigma_{0}$, by moving the line of integration from $5 / 4$ to $-1 / 2$, the integral is equal to

(9) $\left|\Gamma\left(s_{0}\right)\right|^{2} \prod_{p \mid q}\left(1-\frac{1}{p^{2 \sigma_{0}-k+1}}\right) \zeta\left(2 \sigma_{0}-k+1\right)$
$+\frac{\prod_{p \mid q}(1-1 / p)(2 \pi)^{2 \sigma_{0}-k}}{k-2 \sigma_{0}} \Gamma\left(k / 2+i t_{0}\right) \Gamma\left(k / 2-i t_{0}\right) x^{k / 2-\sigma_{0}}+O_{\sigma_{0}, q, k}\left(x^{-1 / 2}\right)$.

Now in (7) we estimate the remainder term $R$. We calculate

$$
\sum_{m, n \geq 1} Z\left(\sigma_{0}, m n / x\right)(m, n)^{1 / 2}(m n)^{-\sigma_{0}+k-1} .
$$

It is

$$
\frac{1}{2 \pi i} \int_{((k+1) / 2)}(2 \pi)^{-2 s}\left(\Gamma\left(s+\sigma_{0}\right)\right)^{2} x^{s}\left(\sum_{m, n \geq 1}(m, n)^{1 / 2}(m n)^{-\left(s+\sigma_{0}-k+1\right)}\right) \frac{d s}{s} .
$$

Note that since the integrand does not have any pole in the strip $5 / 4<$ $\operatorname{Re}(s)<(k+1) / 2$, we can move the line of integration from $5 / 4$ to $(k+1) / 2$. From [4], Lemma 4, we know that

$$
\begin{aligned}
\sum_{m, n \geq 1}(m, n)^{1 / 2}(m n)^{-\left(s+\sigma_{0}-k+1\right)} & \\
= & \frac{\zeta\left(2 s+2 \sigma_{0}-2 k+3 / 2\right) \zeta\left(s+\sigma_{0}-k+1\right)^{2}}{\zeta\left(2 s+2 s_{0}-2 k+2\right)} .
\end{aligned}
$$

Applying this identity to the above integral and moving the line of integration from $(k+1) / 2$ to $k-\sigma_{0}-\varepsilon(\varepsilon>0)$ yields

$$
\sum_{m, n \geq 1} Z\left(\sigma_{0}, m n / x\right)(m, n)^{1 / 2}(m n)^{-\left(s+\sigma_{0}-k+1\right)} \sim C_{\sigma_{0}, k} x^{k-\sigma_{0}} \log x
$$

and by (8), $R \ll N^{1 / 2-k} x^{k-\sigma_{0}} \log x$. Therefore we have

$$
\begin{aligned}
\sum_{f \in \mathcal{P}_{N}} \omega_{f} \mathcal{B}_{f}\left(x, s_{0}\right) & \\
= & \left|\Gamma\left(s_{0}\right)\right|^{2} \prod_{p \mid q}\left(1-\frac{1}{p^{2 \sigma_{0}-k+1}}\right) \zeta\left(2 \sigma_{0}-k+1\right) \\
& +\frac{\prod_{p \mid q}(1-1 / p)(2 \pi)^{2 \sigma_{0}-k}}{k-2 \sigma_{0}} \Gamma\left(k / 2+i t_{0}\right) \Gamma\left(k / 2-i t_{0}\right) x^{k / 2-\sigma_{0}} \\
& +O_{\sigma_{0}, q, k}\left(x^{-1 / 2}\right)+O_{\sigma_{0}, k}\left(N^{1 / 2-k} x^{k-\sigma_{0}} \log x\right) .
\end{aligned}
$$


Lemma 7. Let $\chi$ be a fixed primitive Dirichlet character mod $q$ with $(q, N)=1$ and let $s_{0}=\sigma_{0}+i t_{0}$ where $k / 2<\sigma_{0} \leq(k+1) / 2$. Then

$$
\begin{aligned}
\sum_{f \in \mathcal{P}_{N}} \omega_{f} \mid & \left.L_{f}\left(s_{0}, \chi\right)\right|^{2} \\
& =\prod_{p \mid q}\left(1-\frac{1}{p^{2 \sigma_{0}-k+1}}\right) \zeta\left(2 \sigma_{0}-k+1\right)+c_{1} N^{k / 2-\sigma_{0}}+O_{s_{0}, q, k}\left(N^{-1 / 2}\right)
\end{aligned}
$$

for $N$ prime. Here, $c_{1}$ depends on $s_{0}, q$ and $k$.

Proof. Choosing $x=q^{2} N$ in Lemma 6 and applying (11) in it, proves the lemma.

Proposition 2. Under the assumptions of Proposition 1,

$$
\begin{aligned}
\sum_{f \in \mathcal{P}_{N}} \omega_{f}\left|L_{f}\left(s_{f}, \chi\right)\right|^{2}= & \prod_{p \mid q}\left(1-\frac{1}{p^{2 \sigma_{0}-k+1}}\right) \zeta\left(2 \sigma_{0}-k+1\right)+c_{1} N^{k / 2-\sigma_{0}} \\
& +O_{s_{0}, q, k}\left(N^{-1 / 2}\right)+O_{\sigma_{0}, k}\left(\frac{r_{N}}{R-r_{N}}\right) \\
& +O_{s_{0}, q, k}\left(\frac{r_{N}}{R-r_{N}} N^{k / 2-\sigma_{0}+R}\right) .
\end{aligned}
$$

Here, $c_{1}$ depends on $s_{0}, q$ and $k$.

Proof. We have

$$
\begin{aligned}
\left.\left|\sum_{f \in \mathcal{P}_{N}} \omega_{f}\right| L_{f}\left(s_{f}, \chi\right)\right|^{2}-\sum_{f \in \mathcal{P}_{N}} \omega_{f}\left|L_{f}\left(s_{0}, \chi\right)\right|^{2} \mid \\
\leq\left.\sum_{f \in \mathcal{P}_{N}} \omega_{f}|| L_{f}\left(s_{f}, \chi\right)\right|^{2}-\left|L_{f}\left(s_{0}, \chi\right)\right|^{2} \mid \\
\leq \sum_{f \in \mathcal{P}_{N}} \omega_{f}\left|L_{f}^{2}\left(s_{f}, \chi\right)-L_{f}^{2}\left(s_{0}, \chi\right)\right| .
\end{aligned}
$$

By applying Cauchy's integral formula and Lemma 7, the last expression equals to

$$
\begin{aligned}
\sum_{f \in \mathcal{P}_{N}} \omega_{f} \mid \frac{1}{2 \pi i} \int_{\Gamma} L_{f}^{2}(w, \chi) & \frac{s_{f}-s_{0}}{\left(w-s_{f}\right)\left(w-s_{0}\right)} d w \mid \\
& \leq \frac{r_{N}}{R-r_{N}}\left(O_{\sigma_{0}, k}(1)+O_{s_{0}, q, k}\left(N^{k / 2-\sigma_{0}+R}\right)\right) .
\end{aligned}
$$

This shows that 


$$
\begin{aligned}
& \sum_{f \in \mathcal{P}_{N}} \omega_{f}\left|L_{f}\left(s_{f}, \chi\right)\right|^{2} \\
& =\sum_{f \in \mathcal{P}_{N}} \omega_{f}\left|L_{f}\left(s_{0}, \chi\right)\right|^{2}+\frac{r_{N}}{R-r_{N}}\left(O_{\sigma_{0}, k}(1)+O_{s_{0}, q, k}\left(N^{k / 2-\sigma_{0}+R}\right)\right) .
\end{aligned}
$$

Now applying Lemma 7 in (12) completes the proof.

5. Proof of Theorem 1. We need the following estimation of $\omega_{f}$.

Proposition 3. For $N$ prime we have

$$
\omega_{f} \ll_{k} \begin{cases}(\log N) / N, & f \in \mathcal{F}_{N}, \\ 1 / N, & f \in \mathcal{P}_{N}-\mathcal{F}_{N} .\end{cases}
$$

Pro of. See [4], Proposition 4, for the case $f \in \mathcal{F}_{N}$. If $f \in \mathcal{P}_{N}-\mathcal{F}_{N}$ then

$$
f(z)=h(z) \pm N^{k / 2} h(N z)
$$

as mentioned in the proof of Lemma 1. Now the result follows from the fact that

$$
\langle f, f\rangle=\left\langle h(z) \pm N^{k / 2} h(N z), h(z) \pm N^{k / 2} h(N z)\right\rangle
$$

is bounded below by a constant multiple of $N$ (see [1], Proposition 5.3 for the details).

Now we can prove our theorem. Set

$$
\mathcal{E}_{N}=\left\{f \in \mathcal{P}_{N}: L_{f}(s, \chi) \neq 0 \text { for all } s \text { in } C_{N}\right\} .
$$

Proposition 1 shows that $\mathcal{E}_{N} \neq \emptyset$ for large $N$. Now if $f \in \mathcal{P}_{N}-\mathcal{E}_{N}$ we choose $s_{f}$ such that $L_{f}\left(s_{f}, \chi\right)=0$. With this choice of $s_{f}$ for elements of $\mathcal{P}_{N}-\mathcal{E}_{N}$ and arbitrary choice of $s_{f}$ in $C_{N}$ for elements of $\mathcal{E}_{N}$ and applying the Cauchy-Schwarz inequality and (13), we get

$$
\begin{aligned}
\left|\sum_{f \in \mathcal{P}_{N}} \omega_{f} L_{f}\left(s_{f}, \chi\right)\right|^{2} & \left|\sum_{f \in \mathcal{E}_{N}} \omega_{f} L_{f}\left(s_{f}, \chi\right)\right|^{2} \\
\leq & \left(\sum_{f \in \mathcal{E}_{N} \cap \mathcal{F}_{N}} \omega_{f}+\sum_{f \in \mathcal{E}_{N}-\mathcal{F}_{N}} \omega_{f}\right)\left(\sum_{f \in \mathcal{P}_{N}} \omega_{f}\left|L_{f}\left(s_{f}, \chi\right)\right|^{2}\right) \\
\ll & \left(\sharp\left\{f \in \mathcal{F}_{N}: L_{f}(s, \chi) \neq 0 \text { for all } s \in C_{N}\right\} \frac{\log N}{N}\right. \\
& \left.+2 \operatorname{dim} S_{k}(1) \frac{1}{N}\right) \sum_{f \in \mathcal{P}_{N}} \omega_{f}\left|L_{f}\left(s_{f}, \chi\right)\right|^{2} .
\end{aligned}
$$

Theorem 1 follows by applying Propositions 1 and 2 in (14). 
6. Proof of Theorem 2. We first establish the analogues of Proposition 1, Lemma 7 and Proposition 2 for a point $s_{0}$ on the critical line $\sigma=k / 2$.

Proposition $1^{\prime}$. Let $N$ be prime, and let $\Gamma$ and $C_{N}$ be the circles with center $\left(k / 2, t_{0}\right)$ and radius $R_{N}$ and $r_{N}$ respectively. Suppose that $0<r_{N}<$ $R_{N}<1 / 2$, and

Then

$$
\frac{r_{N}}{R_{N}} N^{R_{N}}(\log N)^{R_{N}}=o\left(\frac{N^{1 / 2}}{\log N}\right) .
$$

$$
\begin{aligned}
\sum_{f \in \mathcal{P}_{N}} \omega_{f} L_{f}\left(s_{f}, \chi\right)=1 & +O_{q, k}\left(\frac{1}{\Gamma\left(k / 2+t_{0}\right)} N^{-1 / 2} \log N\right) \\
& +O_{q, k, t_{0}}\left(\frac{r_{N}}{R_{N}-r_{N}} N^{R_{N}-1 / 2}(\log N)^{R_{N}+1}\right)
\end{aligned}
$$

where $s_{f}$ is an arbitrary point in $C_{N}$.

Proof. It is similar to the proof of Proposition 1.

Lemma $7^{\prime}$. Let $\chi$ be a fixed primitive Dirichlet character mod $q$ with $(q, N)=1$ and let $s_{0}=k / 2+i t_{0}$. Then

$$
\sum_{f \in \mathcal{P}_{N}} \omega_{f}\left|L_{f}\left(k / 2+i t_{0}, \chi\right)\right|^{2}=\prod_{p \mid q}\left(1-\frac{1}{p}\right) \log N+c_{1}+O_{t_{0}, q, k}\left(N^{-1 / 2} \log N\right)
$$

for $N$ prime. Here, $c_{1}$ depends on $t_{0}, q$ and $k$.

Proof. The proof is exactly similar to the proof of Lemma 7 . The result follows by observing that

$$
\frac{1}{2 \pi i} \int_{(5 / 4)} L\left(2 s+1, \chi_{0}\right)(2 \pi)^{-2 s} \Gamma\left(s+k / 2+i t_{0}\right) \Gamma\left(s+k / 2-i t_{0}\right) x^{s} \frac{d s}{s}
$$

has a double pole at $s=k / 2$ which contributes $\log N$ to the main term (see [1], Proposition 4.2 for the details).

Lemma 8. Let $\Gamma$ be a circle with center $\left(k / 2, t_{0}\right)$ and radius $0<R_{N}$ $<1 / 2$, and let $w$ be a point on (or inside) $\Gamma$. Then if $\sigma=\operatorname{Re}(w) \geq k / 2$,

$$
\sum_{f \in \mathcal{P}_{N}} \omega_{f}\left|L_{f}(w, \chi)\right|^{2} \ll_{k, q, t_{0}}(\log N)^{4}
$$

and if $\sigma=\operatorname{Re}(w) \leq k / 2$,

$$
\sum_{f \in \mathcal{P}_{N}} \omega_{f}\left|L_{f}(w, \chi)\right|^{2} \ll_{k, q, t_{0}} N^{k-2 \sigma}(\log N)^{4} .
$$


Pr o of. First we assume that $\sigma=\operatorname{Re}(w) \geq k / 2$. Choosing $x=q^{2} N \log N$ in Lemma 3 gives

$$
\begin{aligned}
\Gamma(w) L_{f}(w, \chi)= & \sum_{n \geq 1} \frac{\chi(n) a_{f}(n)}{n^{w}} W\left(w, \frac{2 \pi n}{q^{2} N \log N}\right) \\
& +O_{q, k}\left(N^{-6+k / 2-\sigma}(\log N)^{k-\sigma+1}\right) .
\end{aligned}
$$

Now by applying the upper bound of Lemma 1 for $a_{f}(n)$ and the upper bound of Lemma 4 for $W(w, \cdot)$, we deduce that

$$
\sum_{n>q^{2} N(\log N)^{2}} \frac{\chi(n) a_{f}(n)}{n^{w}} W\left(w, \frac{2 \pi n}{q^{2} N \log N}\right)=O_{q, k}\left(N^{-5+k / 2-\sigma}(\log N)^{k-\sigma}\right) .
$$

Therefore

$$
\begin{aligned}
& \Gamma(w) L_{f}(w, \chi) \\
& =\sum_{n \leq q^{2} N(\log N)^{2}} \frac{\chi(n)}{n^{w}} W\left(w, \frac{2 \pi n}{q^{2} N \log N}\right) a_{f}(n)+O_{q, k}\left(N^{-5}(\log N)^{k / 2}\right) .
\end{aligned}
$$

We know that for complex numbers $c_{n}$,

$$
\sum_{f \in \mathcal{P}_{N}} \omega_{f}\left|\sum_{n \leq X} c_{n} a_{f}(n)\right|^{2}=\left(1+O\left(N^{-1} X \log X\right)\right) \sum_{n \leq X} n^{k-1}\left|c_{n}\right|^{2}
$$

with an absolute implied constant (see [5], Theorem 1). Applying this identity for

$$
X=N q^{2}(\log N)^{2}, \quad c_{n}=\frac{\chi(n)}{n^{w}} W\left(w, \frac{2 \pi n}{q^{2} N \log N}\right),
$$

and using Lemma 4 imply that

$$
\begin{aligned}
\sum_{f \in \mathcal{P}_{N}} \omega_{f}\left|\sum_{n \leq q^{2} N(\log N)^{2}} c_{n} a_{f}(n)\right|^{2} & =O_{q, k}\left((\log N)^{3} \sum_{n \leq q^{2} N(\log N)^{2}} \frac{1}{n^{2 \sigma-k+1}}\right) \\
& =O_{q, k}\left((\log N)^{4}\right) .
\end{aligned}
$$

This together with (15) proves the lemma.

If $\sigma=\operatorname{Re}(w)<k / 2$ the assertion results from the functional equation of $\left|L_{f}(w, \chi)\right|^{2}$.

Proposition $2^{\prime}$. Let $N$ be prime, and let $\Gamma$ and $C_{N}$ be the circles with center $\left(k / 2, t_{0}\right)$ and radius $R_{N}$ and $r_{N}$ respectively. Suppose that $0<r_{N}<$ $R_{N}<1 / 2$ and

$$
\frac{r_{N} N^{2 R_{N}}}{R_{N}}=o\left(\frac{1}{(\log N)^{3}}\right)
$$


Then

$$
\begin{aligned}
\sum_{f \in \mathcal{P}_{N}} \omega_{f}\left|L_{f}\left(s_{f}, \chi\right)\right|^{2}= & \prod_{p \mid q}\left(1-\frac{1}{p}\right) \log N+c_{1} \\
& +O_{t_{0}, q, k}\left(N^{-1 / 2} \log N\right)+O_{t_{0}, q, k}\left(\frac{r_{N} N^{2 R_{N}}(\log N)^{4}}{R_{N}-r_{N}}\right)
\end{aligned}
$$

where $s_{f}$ is an arbitrary point in $C_{N}$ and $c_{1}$ depends on $t_{0}, q$ and $k$.

Pr o of. From the proof of Proposition 2, we know that

$$
\begin{aligned}
\sum_{f \in \mathcal{P}_{N}} \omega_{f}\left|L_{f}\left(s_{f}, \chi\right)\right|^{2}= & \sum_{f \in \mathcal{P}_{N}} \omega\left|L_{f}(k / 2+i t, \chi)\right|^{2} \\
& +\sum_{f \in \mathcal{P}_{N}} \omega_{f}\left|\frac{1}{2 \pi i} \int_{\Gamma} L_{f}^{2}(w, \chi) \frac{s_{f}-s_{0}}{\left(w-s_{f}\right)\left(w-s_{0}\right)} d \omega\right| .
\end{aligned}
$$

The result follows by applying Lemma $7^{\prime}$ in the above identity and the fact that by Lemma 8 ,

$$
\begin{aligned}
\sum_{f \in \mathcal{P}_{N}} \omega_{f}\left|\frac{1}{2 \pi i} \int_{\Gamma} L_{f}^{2}(w, \chi) \frac{s_{f}-s_{0}}{\left(w-s_{f}\right)\left(w-s_{0}\right)} d \omega\right| \\
\leq \frac{r_{N}}{R_{N}-r_{N}} O_{t_{0}, q, k}\left(N^{2 R_{N}}(\log N)^{4}\right)
\end{aligned}
$$

Now in Propositions $1^{\prime}$ and $2^{\prime}$, let $R_{N}=1 / \log N$ and $r_{N}=1 /(\log N)^{4+\varepsilon}$. We then proceed in a way similar to the proof of Theorem 1 and finally Theorem 2 follows by applying Propositions $1^{\prime}$ and $2^{\prime}$ in (14).

\section{References}

[1] A. Akbary, Non-vanishing of weight $k$ modular L-functions with large level, J. Ramanujan Math. Soc. 14 (1999), 37-54.

[2] R. Balasubramanian, A note on Dirichlet's L-functions, Acta Arith. 38 (1980), 273-283.

[3] R. Balasubramanian and V. K. Murty, Zeros of Dirichlet L-functions, Ann. Sci. École. Norm. Sup. 25 (1992), 567-615.

[4] W. Duke, The critical order of vanishing of automorphic L-functions with large level, Invent. Math. 119 (1995), 165-174.

[5] W. Duke, J. B. Friedlander and H. Iwaniec, Bounds for automorphic L-functions. II, ibid. 115 (1994), 219-239.

[6] P. D. T. A. Elliott, On the distribution of the values of Dirichlet L-series in the half plane $\sigma>1 / 2$, Indag. Math. 33 (1971), 222-234.

[7] V. K. Murty and T. Stefanicki, Average values of quadratic twists of modular $L$-functions, unpublished.

[8] A. Perelli and J. Pomykała, Averages of twisted elliptic L-functions, Acta Arith. 80 (1997), 149-163. 
[9] A. Pizer, Hecke operators for $\Gamma_{0}(N)$, J. Algebra 83 (1983), 39-64.

Department of Mathematics and Statistics

Concordia University

1455 de Maisonneuve Blvd. West

Montréal, Quebec, H3G 1M8

Canada

E-mail: akbary@cicma.concordia.ca

Received on 28.4.1998

and in revised form on 10.12.1999 